\title{
Searching for lepton flavor violating interactions at future electron-positron colliders
}

\author{
S. M. Etesami, R. Jafari®, M. Mohammadi Najafabadi®, and S. Tizchang $\odot$ \\ School of Particles and Accelerators, Institute for Research in Fundamental Sciences (IPM), \\ P.O. Box 19395-5531, Tehran, Iran
}

(Received 5 March 2021; accepted 18 June 2021; published 30 July 2021)

\begin{abstract}
Lepton flavor violating interactions are absent in the standard model but are expected in various beyondstandard models. In this work, the potential of the future circular electron-positron collider to probe the four-fermion lepton flavor couplings via the $e^{+} e^{-} \rightarrow e^{ \pm} \tau^{\mp}$ process is revisited by means of an effective field theory approach. We provide constraints at $95 \%$ C.L. on the dimension-six Wilson coefficients including major sources of background processes and considering realistic detector effects at four expected operation energies, $\sqrt{s}=157.5,162.5,240$, and $365 \mathrm{GeV}$, according to their corresponding integrated luminosities. We demonstrate that the statistical combination of the results from four center-of-mass energies improves the sensitivity to the lepton flavor violation couplings significantly. We compare the results with the prospects from the Belle II Collaboration with $50 \mathrm{ab}^{-1}$ and other studies at electronpositron colliders.
\end{abstract}

DOI: $10.1103 /$ PhysRevD.104.015034

\section{INTRODUCTION}

In the standard model (SM) with massless neutrinos, processes with lepton flavor violation (LFV) interactions are forbidden [1]. However, experimental observations of neutrino oscillations show that neutrinos are massive and mix with each other which leads to the violation of lepton flavor conservation [2]. LFV enters into the charged lepton sector from the neutrino sector via radiative corrections which are extremely suppressed because of the smallness of the ratio of neutrino mass to the $\mathrm{W}$ boson mass $[3,4]$. The predicted branching fraction, for example, for $\tau^{-} \rightarrow \ell^{+} \ell^{-} \ell^{\prime-}$ decays are approximately $\lesssim 10^{-54}$, where $\ell=e, \mu$ [4]. However, an increase of several orders of magnitude is predicted in some extensions of the SM, such as the supersymmetric SM [5-7], resulting in branching fractions observable in experiments. Therefore, any observation of LFV in the charged lepton sector would be an obvious hint to the presence of physics beyond the SM, and can be an indirect way to search for beyond-the-SM scenarios.

So far, no LFV interactions among the charged leptons have been observed and there are several strong constraints from various experiments. For instance, $\ell^{-} \rightarrow e^{-} e^{+} e^{-}$, $\ell \rightarrow e \gamma$ with $\ell=\tau, \mu$, rare decays of mesons, $Z$ boson decays, Higgs boson decays, and heavy resonances have

Published by the American Physical Society under the terms of the Creative Commons Attribution 4.0 International license. Further distribution of this work must maintain attribution to the author(s) and the published article's title, journal citation, and DOI. Funded by SCOAP ${ }^{3}$. been used to probe LFV in different experiments [8-17]. The most stringent bounds at $90 \%$ C.L. on the LFV decays of the $\tau$ leptons into $3 e$ were measured by BABAR and Belle Collaborations $[18,19]$ :

$$
\begin{aligned}
& \mathcal{B}\left(\tau^{-} \rightarrow e^{-} e^{+} e^{-}\right) \leq 2.9 \times 10^{-8}(\text { BABAR }), \\
& \mathcal{B}\left(\tau^{-} \rightarrow e^{-} e^{+} e^{-}\right) \leq 2.7 \times 10^{-8}(\text { Belle })
\end{aligned}
$$

The Belle II future prospects for the upper limit on $\mathcal{B}\left(\tau^{-} \rightarrow\right.$ $\left.e^{-} e^{+} e^{-}\right)$at $90 \%$ C.L., assuming the integrated luminosity of $50 \mathrm{ab}^{-1}$, is $\lesssim 10^{-10}$ [20].

The proposed future lepton colliders, such as the international linear collider (ILC Collaboration) [21-23], the compact linear collider (CLIC Collaboration) [24-26], the circular electron-positron collider (CPEC Collaboration) $[27,28]$, and the future circular collider with electronpositron collisions with highest luminosity (FCC-ee Collaboration) [29], are expected to provide an extraordinary place to perform flavor physics studies. There are a variety of theories that give rise to LFV. For instance, the additional fermions present in the type III seesaw model, or in the lowscale seesaw models, give rise to large LFV effects [30-32]. The LFV through $Z$, the Higgs boson, and other new degrees of freedom have been studied in Refs. [33-36], and the Higgs and scalar LFV decays have been presented in Refs. [37-40]. If the new degrees of freedom contributing to LFV are heavy compared to the energy accessible at colliders, then the LFV couplings could be reasonably parametrized via the effective contact interactions. Experiments can perfectly search for the LFV in a model-independent approach, without any 
theoretical input. Effective field theories allow for a modelindependent interpretation of the experimental results. However, in a true bottom-up approach, all relevant operators have to be considered since no symmetry or model consideration is present to suppress some operators with respect to other operators.

LFV $\bar{e} e \bar{e} \tau$ contact interactions have been already studied at future high energy lepton colliders through $e^{+} e^{-} \rightarrow$ $e^{ \pm} \tau^{\mp}$ in Refs. [41,42]. In Ref. [42], the LFV contact operators probed via $e^{+} e^{-} \rightarrow e^{ \pm} \tau^{\mp}$ the process at $\sqrt{s}=250,500,1000,3000 \mathrm{GeV}$ considering two main background sources, $\tau^{+} \tau^{-}$and $e \tau \nu_{e} \nu_{\tau}$. A similar process was examined at $\sqrt{s}=250,500,1000 \mathrm{GeV}$ in Ref. [42] where the effects of polarization of the electron and positron beam have been investigated. The detector response has been simulated using the DELPHES package [43] and $e^{ \pm} \tau^{\mp} \nu_{e} \nu_{\tau}$ has been considered the main source of background.

The purpose of this paper is to investigate the LFV contact interactions of $e \bar{e} e \bar{\tau}$ at a future lepton collider. In particular, the focus is on a model-independent search for various types of four-lepton LFV interactions leading to the production of $e^{ \pm} \tau^{\mp}$ at the future circular electron-positron collider. The search is performed at the proposed energies and integrated luminosity benchmarks of the FCC-ee. Particularly, the analysis is carried out at the center-of-mass energies of $157.5,162.5,240$, and $365 \mathrm{GeV}$ with the corresponding integrated luminosities of 5, 5, 5, and $1.5 \mathrm{ab}^{-1}$, respectively. A realistic detector response is taken into account using the DELPHES package. The main sources of background processes are $\tau^{+} \tau^{-}, e^{ \pm} \tau^{\mp} \nu_{e} \nu_{\tau}, \ell^{ \pm} \ell^{\mp} \ell^{\prime \pm} \ell^{\prime \mp}, \ell^{ \pm} \ell^{\mp} j j, \ell \nu j j$, $(\ell=e, \mu, \tau)$, and $j j,(j=$ jet $)$. Finally, a statistical combination is performed over the results obtained at the four center-of-mass energies.

The layout of this paper is as follows. In Sec. II the effective operators describing LFV are briefly presented. Section III is dedicated to presenting the simulation details and analysis strategy. In Sec. IV, the constraints on LFV couplings and the results of statistical combinations are given. Finally, Sec. V concludes the paper.

\section{THE LFV EFFECTIVE LAGRANGIAN}

For studying the $\bar{e} e \bar{e} \tau \mathrm{LFV}$ couplings, it is customary in the literature to consider four-fermi contact interactions which provide the opportunity to characterize the new physics effects in a model-independent framework. In general, there is a set of six chirality conserving scalar and vector form four-fermi operators with $\Delta L=1$ where $\Delta L$ represents the difference between initial and final state lepton numbers [44]. The operators are classified into two types: the scalar type $(S)$ and vector type $(V)$ interactions. In addition, there are LFV operators containing dipole structures which are tightly constrained by radiative LFV decays [45]; therefore, they are not considered in this work.
The effective Lagrangian and the relevant set of operators leading to $e^{ \pm} \tau^{\mp}$ production by either a scalar or a vector are given by [44]:

$$
\begin{aligned}
& \mathcal{L}_{\text {eff }} \supset \sum_{\alpha, \beta} \sum_{i j} \frac{c_{\alpha \beta}^{i j}}{\Lambda^{2}} \mathcal{O}_{\alpha \beta}^{i j}, \\
& \mathcal{O}_{R L}^{S, i j}=\left(\bar{\ell}_{j L} \ell_{i R}\right)\left(\bar{\ell}_{j L} \ell_{j R}\right), \\
& \mathcal{O}_{L R}^{S, i j}=\left(\bar{\ell}_{i R} \ell_{j L}\right)\left(\bar{\ell}_{j R} \ell_{j L}\right), \\
& \mathcal{O}_{R R}^{V, i j}=\left(\bar{\ell}_{i R} \gamma^{\mu} \ell_{j R}\right)\left(\bar{\ell}_{j R} \gamma_{\mu} \ell_{j R}\right), \\
& \mathcal{O}_{L L}^{V, i j}=\left(\bar{\ell}_{i L} \gamma^{\mu} \ell_{j L}\right)\left(\bar{\ell}_{V, j L} \gamma_{\mu} \ell_{j L}\right), \\
& \mathcal{O}_{L R}^{V, i j}=\left(\bar{e}_{i L} \gamma^{\mu} \ell_{j L}\right),\left(\bar{\ell}_{j R} \gamma_{\mu} \ell_{j R}\right), \\
& \mathcal{O}_{R L}^{V, i j}=\left(\bar{\ell}_{i R} \gamma^{\mu} \ell_{j R}\right)\left(\bar{\ell}_{i L} \gamma_{\mu} \ell_{i L}\right),
\end{aligned}
$$

where $\mathcal{O}_{\alpha \beta}^{i j}$ are the four-fermion leptonic operators, $\Lambda$ is the new physics energy scale, and $c_{\alpha \beta}^{i j}$ indicate the effective Wilson coupling between leptons of flavor $i$ and $j$ and $\alpha \beta$ Lorentz structures. The operators are invariant under the SM gauge symmetry $\mathrm{SU}(3) \times \mathrm{SU}(2) \times \mathrm{U}(1)$. It is found that flavor violation among first and second generations of leptons is tightly constrained by experimental constraints arising from muon decay into three electron $\mu \rightarrow$ eee at the SINDRUM experiment [46], the muon transition to $e \gamma$ [47], and $\mu-e$ conversion [48]. However, constraints on flavor violations between electrons and $\tau$ and muons and $\tau$ are much looser. As a consequence, we restrict our study to $\bar{e} e \bar{e} \tau$ couplings using the $e^{-} e^{+} \rightarrow e^{ \pm} \tau^{\mp}$ process.

In addition to the $\bar{e} e \bar{e} \tau$ four-fermi contact interactions, contact interactions among leptons and quarks (like $\bar{e} e \bar{q} q^{\prime}$ ), and electrons and Higgs- $Z$ (eeHZ) are favorite topics which have been probed in several papers such as Refs. [49-52].

In order to see the dependence of the production rate to the center-of-mass energy $\sqrt{s}$ and to find a feeling about the sensitivity to different types of operators, the expression for the cross section of the $e^{-} e^{+} \rightarrow e^{ \pm} \tau^{\mp}$ process is presented. The theoretical cross section of $\sigma\left(e^{-} e^{+} \rightarrow\right.$ $\left.e^{+} \tau^{-}\right)+\sigma\left(e^{-} e^{+} \rightarrow e^{-} \tau^{+}\right)$in the presence of all couplings has the following form [53]:

$$
\begin{aligned}
\sigma(s)= & \frac{s}{96 \pi \Lambda^{4}}\left\{\left(\left|c_{L R}^{S}\right|^{2}+\left|c_{R L}^{S}\right|^{2}\right)+16\left(\left|c_{L L}^{V}\right|^{2}+\left|c_{R R}^{V}\right|^{2}\right.\right. \\
& \left.\left.+\left|c_{L R}^{V}\right|^{2}+\left|c_{R L}^{V}\right|^{2}\right)\right\},
\end{aligned}
$$

where in finding the above expression, the lepton masses are set to zero considering the center-of-mass energy scale. As seen, the production rate of the four-fermion interactions grows linearly with the squared center-of-mass energy $s$, and diverges when $s \rightarrow \infty$. However, one should note that we are working in a nonrenormalizable formalism and that these operators provide an acceptable description 
of physics at high energy up to an energy scale $\Lambda$. Another interesting point which is worth mentioning is that the vector type operators contribute to the LFV production of $e \tau$ which are larger than the scalar type operators by a factor of 16. Therefore, better sensitivity is expected for the vector type with respect to scalar type operators.

In the next section, we describe the simulation method and details of the analysis to search for the LFV operators at four energy benchmarks of the FCC-ee.

\section{SIMULATION DETAILS AND ANALYSIS STRATEGY}

The main goal of this work is to estimate the potential of the proposed FCC-ee collider to probe the effective LFV operators introduced in Eq. (2) using the $e^{-} e^{+} \rightarrow e^{ \pm} \tau^{\mp}$ process. This section is dedicated to presenting event generation, the simulation of detector effects, and the analysis method to find the LFV sensitivity for four energy benchmarks. In particular, the search is separately performed at center-of-mass energies 365, 240, 162.5, and 157.5 GeV with their expected integrated luminosities of $1.5,5,5$, and $5 \mathrm{ab}^{-1}$ at the FCC-ee collider, respectively [29]. The signal process consists of an electron (or a positron) and a $\bar{\tau}$ lepton (or a $\tau$ lepton) which decays hadronically. The main background sources which are taken into account in this study are:

$$
\begin{aligned}
& \text { (I) } e^{-} e^{+} \rightarrow e^{ \pm} \tau^{\mp} \nu \bar{\nu}, \\
& \text { (II) } e^{-} e^{+} \rightarrow \tau^{+} \tau^{-}, \\
& \text {(III) } e^{-} e^{+} \rightarrow \ell^{ \pm} \ell^{\mp} \ell^{\prime \pm} \ell^{\prime \mp}\left(\ell, \ell^{\prime}=e, \mu, \tau\right), \\
& \text { (IV) } e^{-} e^{+} \rightarrow \ell^{ \pm} \ell^{\mp} j j(\ell=e, \mu, \tau), \\
& \text { (V) } e^{-} e^{+} \rightarrow \ell^{ \pm} \nu j j(\ell=e, \mu, \tau), \\
& \text { (VI) } e^{-} e^{+} \rightarrow j j .
\end{aligned}
$$

The second background, $\tau^{+} \tau^{-}$, is in particular contributing to the background composition when one of the $\tau$ leptons decay to an electron and the electron is reconstructed in the final state. The third item in the list of backgrounds, $\ell^{ \pm} \ell^{\mp} \ell^{\prime \pm} \ell^{\prime \mp}$, is considered as there is a possibility that two of the leptons are scattered to regions of pseudorapidity where the detector area is blind or two isolated charged leptons are not well reconstructed. Since jets can be misidentified as electrons and also as $\tau$ leptons, the fourth to sixth items must be included in the list of backgrounds to obtain a more realistic assessment of the results.

The effective Lagrangian introduced in Eq. (2) is implemented in the FeynRule program [54] and then the Universal FeynRules Output model [55] is inserted to MadGraph5_aMC@NLO 2.6.6. The events of the signal and backgrounds are generated at leading order with MadGraph5_aMC@NLO 2.6.6 [56-58], including the initial state radiation (ISR) effects. The ISR effects are considered using the MGISR plugin in MadGraph5_aMC@NLO 2.6.6 $[59,60]$. The generated events are passed through PYTHIA 8 $[61,62]$ for the showering, hadronization, and decay of unstable particles. The detector effects are simulated via DELPHES 3.4.2 [43] according to an I-like detector [63]. For electrons with $p_{T}>10 \mathrm{GeV}$ and $|\eta| \leq 2.5$, the identification efficiency in the ILD card is $95 \%$. The electron energy resolution is $\frac{\Delta E}{E}=\frac{0.15}{\sqrt{E}}+0.01$. To calculate the cross section of the signal and backgrounds and simulate the events, the SM relevant input values are set as follows: $M_{Z}=91.188 \mathrm{GeV}$, mass of $\tau$ lepton $m_{\tau}=1.777 \mathrm{GeV}$, $G_{F}=1.166 \times 10^{-5} \mathrm{GeV}^{-2}, \alpha_{e}=1 / 127.9$, and $\alpha_{s}=0.118$.

The $\tau$ lepton lifetime is $\sim 290$ femto/second, corresponding to $c \tau \sim 87 \mu \mathrm{m}$. The actual decay length is obtained by multiplying $c \tau$ with $\beta \gamma$ and therefore a $\tau$ lepton with $E=40 \mathrm{GeV}$ travels around $2 \mathrm{~mm}$ through the detector then decays. In around two-thirds of decays, $\tau s$ decay hadronically, typically to one or three charged mesons (mostly $\pi^{-} \pi^{+}$), often associated with neutral pions, and a $\tau$ neutrino. The $\tau$ tagging efficiency in the ILD simulation card is $40 \%$ and the $\tau$ misidentification rate is assumed to be equal to $0.1 \%$ [64].

Six different signal samples corresponding to the six operators presented in Eq. (3) are generated. In order to generate signal events, we set the related effective Wilson coefficient $c_{i j}=0.1$ with $i=j=L, R$ and $\Lambda=1 \mathrm{TeV}$ and require preselection cuts as $p_{T}^{\ell}=10 \mathrm{GeV}$, the pseudorapidity of leptons is $\left|\eta^{\ell}\right|<3.0$, and the angular separation between two leptons is $\Delta R=\sqrt{(\Delta \eta)^{2}+(\Delta \phi)^{2}}>0.3$. For generating the background samples, we require the addition of angular separation between leptons and jets, that the two jets in the final state be $\Delta R_{\ell, j}>0.3$, and that the $p_{T}=$ $10 \mathrm{GeV}$ and $|\eta|<3.0$ are applied on jets in the final state at generator level.

The cross section and corresponding uncertainties with the ISR effect of backgrounds as well as signals are given in Table I for all center-of-mass energies: 157.5, 162.5, 240, and $365 \mathrm{GeV}$.

The signal events are selected according to the following requirements. We ask for exactly two leptons with opposite charge, one isolated electron, and one $\tau$-tagged lepton. It is required that $p_{T}^{e}>10 \mathrm{GeV}$, that $p_{T}^{\tau}>20 \mathrm{GeV}$, that the pseudorapidity of both leptons satisfies $\left|\eta^{\ell}\right|<2.5$, and that $\Delta R_{e, \tau}>0.5$. In order to make sure the electron candidate is well isolated, it is required that RelIso $<0.15$, where RelIso is defined as the ratio of the sum of $p_{\mathrm{T}}$ of the charged particle tracks inside a cone of size 0.5 around the electron track to the $p_{\mathrm{T}}$ of the electron. $\tau$ leptons which decay via hadronic modes are considered. A $\tau$ lepton in hadronic decay mode produces a jet containing a few neutral and charged hadrons. Therefore, considering the $\tau$ tagging efficiency, a jet is potentially considered to be a $\tau$ candidate if a generated $\tau$ exists within a distance $\Delta R=\sqrt{\left(\eta_{\mathrm{jet}}-\eta_{\tau}\right)^{2}+\left(\phi_{\mathrm{jet}}-\phi_{\tau}\right)^{2}}=0.3$ from the jet axis. 
TABLE I. The cross sections of signal $e^{-} e^{+} \rightarrow e^{ \pm} \tau^{\mp}$ and main background processes with their corresponding uncertainties are presented. The cross section of two signal scenarios are given assuming $c_{L R}^{V}=0.1, c_{L R}^{S}=0.1$, and $\Lambda=1 \mathrm{TeV}$. The cross sections are in the unit of $\mathrm{fb}$ and include the ISR effects.

\begin{tabular}{|c|c|c|c|c|c|c|c|c|}
\hline$\sqrt{s}[\mathrm{GeV}]$ & $c_{L R}^{V}=0.1$ & $c_{L R}^{S}=0.1$ & $e \tau \nu \bar{\nu}$ & $\tau \bar{\tau}$ & $e \bar{\ell} \ell^{\prime} \bar{\ell}^{\prime}$ & $\ell \bar{\ell} j j$ & $\ell \nu j j$ & $j j$ \\
\hline 157.5 & $4.72 \pm 0.007$ & $0.29 \pm 0.0004$ & $22.33 \pm 0.07$ & $11076.5 \pm 3.4$ & $39.86 \pm 0.08$ & $80.95 \pm 0.2$ & $272.9 \pm 0.4$ & $32032 \pm 8.1$ \\
\hline 162.5 & $5.02 \pm 0.007$ & $0.31 \pm 0.0004$ & $102.12 \pm 0.3$ & $10275.8 \pm 2.9$ & $42.23 \pm 0.08$ & $83.06 \pm 0.3$ & $1198.05 \pm 0.8$ & $29133 \pm 6.2$ \\
\hline 240 & $10.98 \pm 0.04$ & $0.69 \pm 0.0008$ & $415.63 \pm 0.6$ & $4196.8 \pm 1.2$ & $86.24 \pm 0.2$ & $217.8 \pm 0.5$ & $4552.7 \pm 1.3$ & $10481 \pm 3.5$ \\
\hline 365 & $25.26 \pm 0.07$ & $1.57 \pm 0.002$ & $327.59 \pm 0.5$ & $1803.6 \pm 0.6$ & $85.05 \pm 0.1$ & $195.13 \pm 0.3$ & $3247.02 \pm 1.1$ & $4306 \pm 1.2$ \\
\hline
\end{tabular}

To suppress the contributions from events with $e^{-} e^{+}$and $\mu^{-} \mu^{+}$in the final state, events that contain two electron or two muon candidates are rejected. This reduces background events from $\ell^{ \pm} \ell^{\mp} \ell^{\prime \pm} \ell^{\prime \mp}, \ell^{ \pm} \ell^{\mp} j j$, and $\tau^{+} \tau^{-}$with $\tau \rightarrow$ $e \nu_{e} \nu_{\tau}$ or $\tau \rightarrow \mu \nu_{\mu} \nu_{\tau}$.

To enhance the sensitivity, we apply additional cuts on the energy of the final state electron and the invariant mass of the two leptons $M_{e \tau}$. Figure 1 displays the distributions of the electron energy in the final state (left plot) and the invariant mass of the final state, i.e., $M_{e \tau}$ (right plot) at a center-of-mass energy of $240 \mathrm{GeV}$. Obviously, both distributions show a significant difference between the shape of the signal and background processes. The $M_{e \tau}$ distributions for the $\ell^{ \pm} \ell^{\mp} j j, \tau^{+} \tau^{-}$, and $\ell^{ \pm} \ell^{\mp} \ell^{\prime \pm} \ell^{\prime \mp}$ background processes peak at lower values with respect to the signal and even the other backgrounds. This is expected as the center-of-mass energy is shared among at least four particles in $\ell^{ \pm} \ell^{\mp} j j$ and $\ell^{ \pm} \ell^{\mp} \ell^{\prime \pm} \ell^{\prime \mp}$ processes. Therefore, cutting on $M_{e \tau}$ effectively suppresses these reducible backgrounds. It is notable that the $M_{e \tau}$ distribution has different behaviors for $c_{L L}^{V}$ and $c_{L R}^{V}$, which arises from the fact that for the $L L$ coupling $d \sigma / d \cos \theta \propto(1+\cos \theta)^{2}$ while for the $L R$ coupling $d \sigma / d \cos \theta \propto(1-\cos \theta)^{2}$. For the signal events, the energy distribution of the electron is expected to spread around $\sqrt{s} / 2$. As a result, it is used to

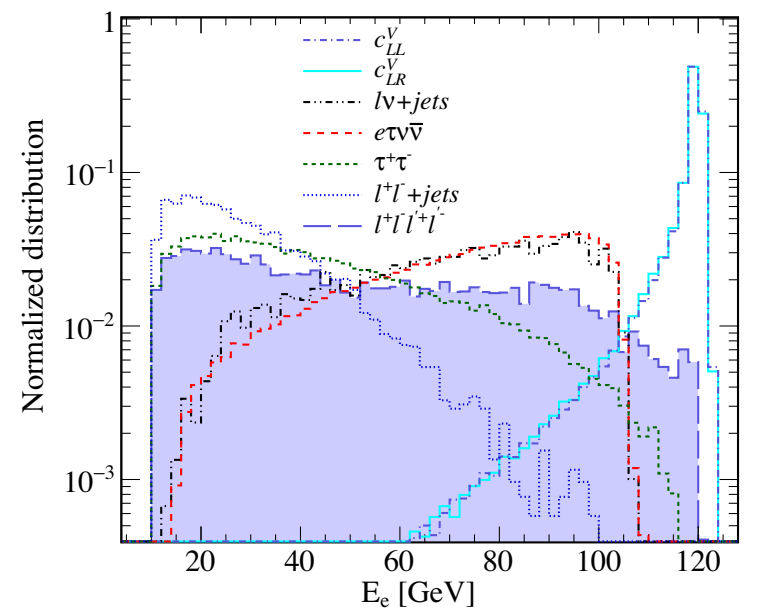

further reduce the contributions of the background processes.

The cut values on $E_{e}$ and $M_{e \tau}$ are optimized such that the best limit on the LFV coefficients are obtained. In order to optimize the cuts on the invariant mass and energy of the outgoing electron, the upper bound on the signal cross section for different cut values on $M_{e \tau}$ and $E_{e}$ is obtained. The values which give the lowest upper bound on the signal cross section are chosen as the optimum values. The cuts on the invariant mass of the final state $M_{e \tau}$ are found to be greater than 65,100 , and $150 \mathrm{GeV}$ for $\sqrt{s}=157.5-162.5$, 240 and $365 \mathrm{GeV}$, respectively. The optimized lower cuts on the energy of the electron are obtained to be 78.6, 81.0, 119.7, and $182.0 \mathrm{GeV}$ for $\sqrt{s}=157.5,162.5,240$, and $365 \mathrm{GeV}$, respectively. Table II presents the efficiencies of the signal with $c_{L R}^{V}=0.1, c_{L R}^{S}=0.1$, and the main SM backgrounds after preselection cuts $M_{e \tau}$ and $E_{e}$ with $\sqrt{s}=157.5,162.5,240$, and $365 \mathrm{GeV}$. After applying the cuts, the main background contributions arise from $\ell^{ \pm} \ell^{\mp} \ell^{\prime \pm} \ell^{\prime \mp}, \tau^{+} \tau^{-}$, and $\ell^{ \pm} \ell^{\mp} j j$, and the rest are remarkably suppressed.

As previously indicated, in the detector, hadronic jets could be misidentified as the $\tau$-leptons or as the electrons, therefore processes with hadronic jets in the final state contribute to the background. The detectors proposed for the future lepton colliders are expected to have a great

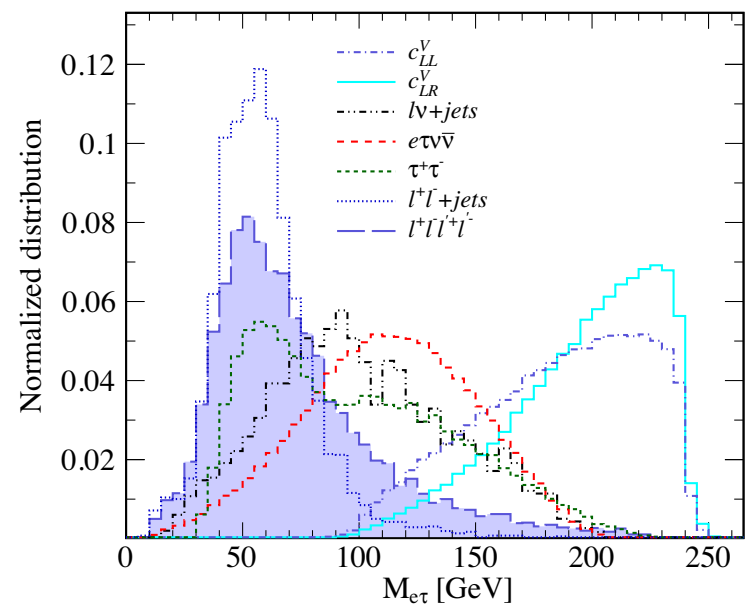

FIG. 1. Normalized distributions of electron energy (left) and the invariant mass of two final state charged leptons $M_{e \tau}$ (right) are presented for the signal benchmarks $c_{L L}^{V}=0.1$ and $c_{R L}^{V}=0.1$, at $\sqrt{s}=240 \mathrm{GeV}$. 
TABLE II. The efficiencies for the signal with $c_{L R}^{V}=0.1, c_{L R}^{S}=0.1$ and the SM backgrounds after selection cuts with $\sqrt{s}=157.5$, $162.5,240$, and $365 \mathrm{GeV}$ are given.

\begin{tabular}{|c|c|c|c|c|c|c|c|}
\hline \multirow[b]{2}{*}{$\sqrt{s}=157.5 \mathrm{GeV}$} & \multicolumn{2}{|c|}{ Signal } & \multicolumn{5}{|c|}{ SM Backgrounds } \\
\hline & $c_{L R}^{V}=0.1$ & $c_{L R}^{S}=0.1$ & $e \tau \nu \bar{\nu}$ & $\tau \bar{\tau}$ & $e \bar{l} \ell^{\prime} \bar{\ell}^{\prime}$ & $\bar{\ell}_{j j}$ & $\ell \nu j j$ \\
\hline (I): Pre-selection cuts & 0.1746 & 0.1698 & 0.099 & 0.045 & $4.9 \times 10^{-3}$ & $1.4 \times 10^{-3}$ & $3.3 \times 10^{-4}$ \\
\hline (II): $M_{e \tau}>65 \mathrm{GeV}$ & 0.1741 & 0.1697 & 0.038 & 0.019 & $2.2 \times 10^{-3}$ & $1.8 \times 10^{-4}$ & $7.5 \times 10^{-5}$ \\
\hline (III): $E_{e}>78.6 \mathrm{GeV}$ & 0.0984 & 0.0831 & $2.8 \times 10^{-8}$ & $1.5 \times 10^{-7}$ & $6.02 \times 10^{-6}$ & $1.7 \times 10^{-7}$ & 0.0 \\
\hline
\end{tabular}

\begin{tabular}{|c|c|c|c|c|c|c|c|}
\hline \multirow[b]{2}{*}{$\sqrt{s}=162.5 \mathrm{GeV}$} & \multicolumn{2}{|c|}{ Signal } & \multicolumn{5}{|c|}{ SM Backgrounds } \\
\hline & $c_{L R}^{V}=0.1$ & $c_{L R}^{S}=0.1$ & $e \tau \nu \bar{\nu}$ & $\tau \bar{\tau}$ & $\bar{\ell} \bar{\ell} \ell^{\prime} \bar{\ell}^{\prime}$ & $\ell \bar{\ell} j j$ & $\ell \nu j j$ \\
\hline (I): Pre-selection cuts & 0.1727 & 0.1711 & 0.106 & 0.048 & $4.9 \times 10^{-3}$ & $1.6 \times 10^{-3}$ & $4.5 \times 10^{-4}$ \\
\hline (II): $M_{e \tau}>65 \mathrm{GeV}$ & 0.1727 & 0.1710 & 0.041 & 0.025 & $2.4 \times 10^{-3}$ & $2.1 \times 10^{-4}$ & $1.0 \times 10^{-4}$ \\
\hline (III): $E_{e}>81 \mathrm{GeV}$ & 0.1122 & 0.0949 & $6 \times 10^{-8}$ & $2.0 \times 10^{-7}$ & $3.61 \times 10^{-6}$ & $2.1 \times 10^{-7}$ & 0.0 \\
\hline
\end{tabular}

\begin{tabular}{|c|c|c|c|c|c|c|c|}
\hline \multirow[b]{2}{*}{$\sqrt{s}=240 \mathrm{GeV}$} & \multicolumn{2}{|c|}{ Signal } & \multicolumn{5}{|c|}{ SM Backgrounds } \\
\hline & $c_{L R}^{V}=0.1$ & $c_{L R}^{S}=0.1$ & $e \tau \nu \bar{\nu}$ & $\tau \bar{\tau}$ & $\ell \bar{l} \ell^{\prime} \bar{\ell}^{\prime}$ & $\ell \bar{l}_{j j}$ & $\ell \nu j j$ \\
\hline (I): Pre-selection cuts & 0.2156 & 0.2137 & 0.131 & 0.037 & $8.8 \times 10^{-3}$ & $6.2 \times 10^{-3}$ & $4.9 \times 10^{-4}$ \\
\hline (II): $M_{e \tau}>100 \mathrm{GeV}$ & 0.2150 & 0.2134 & 0.084 & 0.017 & $1.6 \times 10^{-3}$ & $2.4 \times 10^{-4}$ & $2.0 \times 10^{-4}$ \\
\hline (III): $E_{e}>119.7 \mathrm{GeV}$ & 0.1072 & 0.0989 & $2.1 \times 10^{-8}$ & $1.5 \times 10^{-7}$ & $1.2 \times 10^{-5}$ & $2.4 \times 10^{-7}$ & 0.0 \\
\hline
\end{tabular}

\begin{tabular}{|c|c|c|c|c|c|c|c|}
\hline \multirow[b]{2}{*}{$\underline{\sqrt{s}}=365 \mathrm{GeV}$} & \multicolumn{2}{|c|}{ Signal } & \multicolumn{5}{|c|}{ SM Backgrounds } \\
\hline & $c_{L R}^{V}=0.1$ & $c_{L R}^{S}=0.1$ & $e \tau \nu \bar{\nu}$ & $\tau \bar{\tau}$ & $\bar{\ell} \bar{\ell} \ell^{\prime} \bar{l}^{\prime}$ & $e \bar{\ell} j j$ & $\ell \nu j j$ \\
\hline (I): Pre-selection cuts & 0.2093 & 0.2097 & 0.133 & 0.066 & 0.012 & $6.0 \times 10^{-3}$ & $5.0 \times 10^{-4}$ \\
\hline (II): $M_{e \tau}>150 \mathrm{GeV}$ & 0.2053 & 0.2051 & 0.093 & 0.041 & $2.0 \times 10^{-3}$ & $1.5 \times 10^{-4}$ & $2.4 \times 10^{-4}$ \\
\hline (III): $E_{e}>182 \mathrm{GeV}$ & 0.0993 & 0.0986 & $2.6 \times 10^{-8}$ & $3.2 \times 10^{-7}$ & $2.6 \times 10^{-5}$ & $1.4 \times 10^{-7}$ & 0.0 \\
\hline
\end{tabular}

performance, better than the current modern multipurpose detectors such as ATLAS and CMS detectors. The jet fake $\tau$ probability is expected to be $0.1 \%$ [64]. The rate of background containing jets varies with the center-of-mass energy and is assessed to be less than 5\% of the total background contributions after all selection criteria.

In the next section, we evaluate the potential sensitivities to LFV operators at four energy benchmarks. In addition, a statistical combination of four center-of-mass energies is presented.

\section{RESULTS}

The C.L.s technique $[65,66]$ is exploited to find upper limits on the signal cross section at $95 \%$ C.L. The limits on the signal rates are then translated into the upper limits on the LFV couplings. In the C.L.s method, we define log-likelihood functions $L_{\mathrm{Bkg}}$ and $L_{\mathrm{Signal}+\mathrm{Bkg}}$ for the background hypothesis, and for the signal + background hypothesis as the multiplication of Poissonian likelihood functions. The $p$ value for the hypothesis of signal + background and for the background hypothesis are determined using the log-likelihood ratio $\mathrm{Q}=-2 \ln \left(\mathrm{L}_{\text {signal }+\mathrm{Bkg}} / \mathrm{L}_{\mathrm{Bkg}}\right)$. The signal cross section is constrained using C.L.s $=\mathrm{P}_{\text {signal }+\mathrm{Bkg}}\left(\mathrm{Q}>\mathrm{Q}_{0}\right) /$ $\left(1-\mathrm{P}_{\mathrm{Bkg}}\left(\mathrm{Q}<\mathrm{Q}_{0}\right)\right) \leq 0.05$ which is corresponding to a
95\% confidence level where $\mathrm{Q}_{0}$ is the expected value of the test statistics Q. The Roostats package [67] is used to perform the numerical evaluation of the C.L.s.

The predicted constraints at a 95\% C.L. for $\sqrt{s}=$ 157.5 GeV with $\mathcal{L}=5 \mathrm{ab}^{-1}, \quad \sqrt{s}=162.5 \mathrm{GeV}$ with $\mathcal{L}=5 \mathrm{ab}^{-1}, \sqrt{s}=240 \mathrm{GeV}$ with $\mathcal{L}=5 \mathrm{ab}^{-1}$, and $\sqrt{s}=$ $365 \mathrm{GeV}$ with $\mathcal{L}=1.5 \mathrm{ab}^{-1}$ are presented in Table III. These results are obtained considering only statistical uncertainties and the impacts of systematic and theoretical sources are neglected. For illustration, the results are also given in Fig. 2. As seen, the most sensitivity is achieved on vectortype $L F V$ couplings, i.e., on $c_{L L}^{V}, c_{R R}^{V}, c_{R L}^{V}$, and $c_{L R}^{V}$. This is expected as the production rates for the vector type LFV couplings are larger than the scalar type by a factor of 16. Among various center-of-mass energy scenarios, better sensitivity is expected to be obtained from $\sqrt{s}=$ $365 \mathrm{GeV}$ for which the signal cross section is largest as $\sigma\left(e^{-} e^{+} \rightarrow e \tau\right) \propto s$. Although less data is planned to be collected at $\sqrt{s}=365 \mathrm{GeV}$ with respect to the other energies, a similar sensitivity to other energies at $\sqrt{s}=$ $365 \mathrm{GeV}$ is obtained.

To achieve better sensitivity, the results from four energy benchmarks are combined using the method explained in Ref. [68]. The combined limits are given in Table III and Fig. 2. One can see that the statistical combination of four 
TABLE III. The 95\% C.L. expected upper bounds on the scalar and vector type LFV couplings assuming four center-of-mass energies as well as their combinations are shown. The limits are in the unit of $\mathrm{GeV}^{-2}$. The Belle II future prospects [20], and the expectation from the $e^{-} e^{+}$collider at $\sqrt{s}=1 \mathrm{TeV}$ with polarized beam [42], are presented as well.

\begin{tabular}{|c|c|c|c|c|c|c|}
\hline$\sqrt{s}(\mathrm{GeV}), \mathcal{L}\left(\mathrm{ab}^{-1}\right)$ & $\frac{c_{L L}^{V}}{\Lambda^{2}}\left[\times 10^{-9}\right]$ & $\frac{c_{R R}^{V}}{\Lambda^{2}}\left[\times 10^{-9}\right]$ & $\frac{c_{R L}^{V}}{\Lambda^{2}}\left[\times 10^{-9}\right]$ & $\frac{c_{L R}^{V}}{\Lambda^{2}}\left[\times 10^{-9}\right]$ & $\frac{c_{R L}^{S}}{\Lambda^{2}}\left[\times 10^{-9}\right]$ & $\frac{c_{L R}^{S}}{\Lambda^{2}}\left[\times 10^{-9}\right]$ \\
\hline $157.5,5$ & 5.82 & 5.46 & 5.74 & 5.36 & 21.18 & 22.61 \\
\hline $162.5,5$ & 5.71 & 5.36 & 5.62 & 5.29 & 21.42 & 23.12 \\
\hline 240,5 & 3.69 & 3.50 & 3.73 & 3.53 & 14.81 & 14.74 \\
\hline $365,1.5$ & 3.93 & 3.94 & 3.92 & 3.93 & 15.80 & 15.80 \\
\hline Combination & 1.32 & 1.25 & 1.32 & 1.25 & 5.1 & 5.3 \\
\hline Belle II & 1.06 & 1.06 & 1.55 & 1.55 & 4.29 & 4.29 \\
\hline$\sqrt{s}=1 \mathrm{TeV}$, pol. beam & 4.3 & 1.1 & 1.6 & 1.8 & 13 & 5.9 \\
\hline
\end{tabular}

energy scenarios improves the bounds by almost a factor of around 3 to 4 with respect to the results from a single energy benchmark. It is informative to compare the results from prospects of Belle II with an integrated luminosity of $50 \mathrm{ab}^{-1}$. The limits from combination are competitive with those expected from Belle II. Finally, we compare the results with those which are expected from a future lepton collider at $\sqrt{s}=1 \mathrm{TeV}$ with polarized beams such that $P\left(e^{-}\right)=0.8$ and $P\left(e^{-}\right)=-0.3$ [42]. The results of combination are sensibly better for both the scalar and vector types of the LFV couplings.

In order to have a feeling about the impact of systematic uncertainties on the results, we consider conservative values of uncertainties and re-estimate the sensitivities to the LFV couplings. In Ref. [69], a search for LFV events at LEP2 with the OPAL detector using a similar final state as this work has been performed. The analysis has used the full data collected with OPAL at $\sqrt{s}$ between $189 \mathrm{GeV}$ and $209 \mathrm{GeV}$. The systematic uncertainty on the signal efficiency is $3.5 \%$ and on the number of expected background events is $5 \%$. While for the future experiments the measurements are expected to be made with more accuracy, we consider a conservative value of $5 \%$ uncertainty on both signal selection efficiency and on background expectation. The constraints on $c_{L L}^{V} / \Lambda^{2}, c_{R R}^{V} / \Lambda^{2}, c_{R L}^{V} / \Lambda^{2}, c_{L R}^{V} / \Lambda^{2}$, $c_{R L}^{S} / \Lambda^{2}$, and $c_{L R}^{S} / \Lambda^{2}$, derived at $\sqrt{s}=365 \mathrm{GeV}$, are found
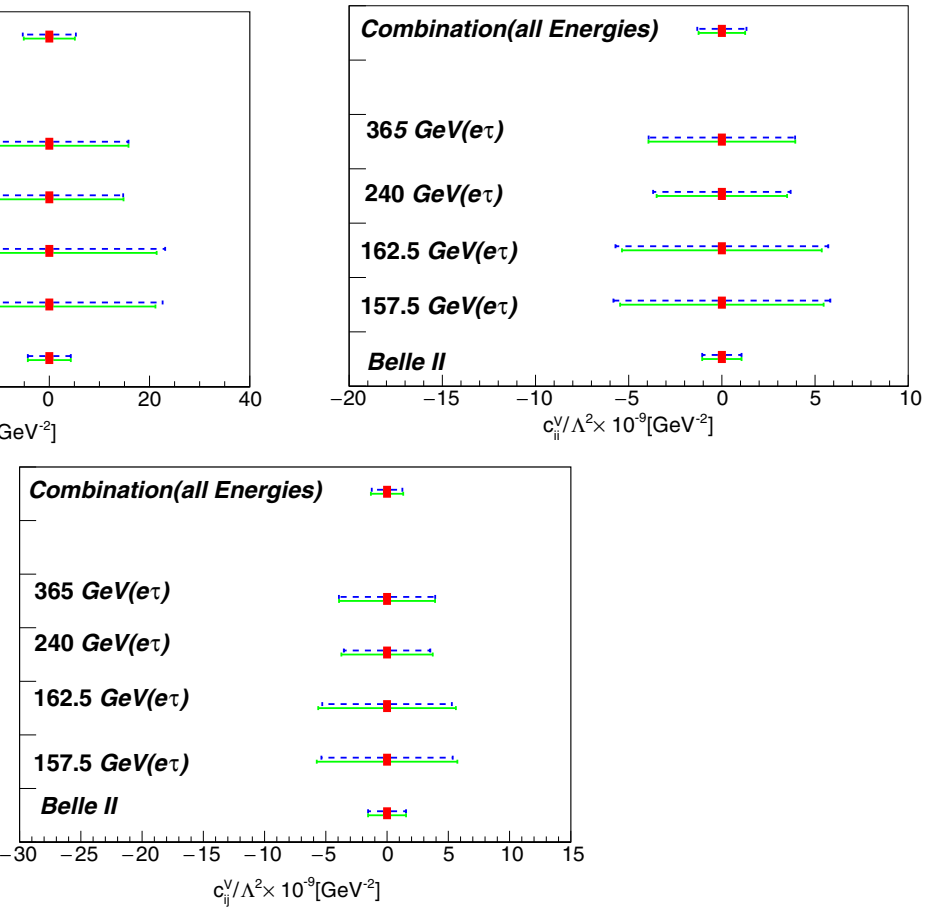

FIG. 2. Expected constraints at $95 \%$ C.L. on $c_{i j}^{S}$ (top left) with $c_{L R}^{S}$ (dashed-blue) and $c_{R L}^{S}$ (solid-green), $c_{i i}^{V}$ (top right) with $c_{L L}^{V}$ (dashed-blue) and $c_{R R}^{V}$ (solid-green), and $c_{i j}^{V}$ (bottom) with $c_{L R}^{V}$ (dashed-blue) and $c_{R L}^{V}$ (solid-green) from $e^{-} e^{+} \rightarrow e \tau$ for four center-ofmass energies are shown. The result of the combination of four energies and the prospects from Belle II with $50 \mathrm{ab}^{-1}$ at $90 \%$ C.L. [20] are presented for comparison. 
to be $4.14 \times 10^{-9}, 4.15 \times 10^{-9}, 4.12 \times 10^{-9}, 4.13 \times 10^{-9}$, $16.57 \times 10^{-9}$, and $16.65 \times 10^{-9} \mathrm{GeV}^{-2}$. Comparing these with the limits without systematic uncertainties shows that including an overall 5\% uncertainty would not weaken the sensitivity remarkably.

\section{SUMMARY AND CONCLUSIONS}

Lepton flavor violation processes are absent in the SM but appear in many extensions of the SM. In particular, the branching fractions of LFV $\tau^{ \pm} \rightarrow \ell^{ \pm} \ell^{ \pm} \ell^{\prime \mp}, \ell, \ell^{\prime}=e, \mu$ decays are increased in various beyond-the-SM scenarios. In this work, the sensitivity of the future circular electronpositron collider, FCC-ee, to probe the LFV couplings is examined using the $e^{-} e^{+} \rightarrow e^{ \pm} \tau^{\mp}$ production. To perform the study effective Lagrangian approaches are exploited; in particular, four-fermi contact interactions with vector and scalar types. In order to find the sensitivity, events are generated for four run scenarios with center-of-mass energies of $157.5,162.5,240$, and $365 \mathrm{GeV}$ with their corresponding benchmarks for the integrated luminosity. The events are generated using MadGraph5_aMC@NLO considering the ISR effect and passed through PYTHIA 8 for the showering, hadronization, and decay of unstable particles. A fast detector simulation is carried out by DELPHES using the ILD detector card. The signal final state consists of an isolated electron and a $\tau$ lepton. In this study, the hadronic decays of the $\tau$ lepton are considered for which the branching fraction is around $64 \%$. Based on the final state, the main sources of reducible and irreducible background processes are taken into account. Cuts on the energy of the final electron and the invariant mass of the $e \tau$ system are applied to suppress the background contributions. Upper limits at 95\% C.L. on the couplings of various types of LFV couplings have been obtained using the C.L.s method for the four center-of-mass energies. Finally, a statistical combination of results obtained at four center-ofmass energies is performed. We show that the statistical combination of four center-of-mass energies increases the sensitivity to the LFV couplings by a factor of two and larger with respect to the limits obtained at $\sqrt{s}=365 \mathrm{GeV}$. The results are compared with those obtained from other studies at lepton colliders considering beam polarizations and with prospects from Belle II. The results of combination are competitive with expectations for Belle II with $50 \mathrm{ab}^{-1}$.

\section{ACKNOWLEDGMENTS}

$\mathrm{S}$. Tizchang is grateful to Qiang $\mathrm{Li}$ for replying the question about implementing ISR in MadGraph5_aMC@NLO. $\mathrm{S}$. M. Etesami is grateful to Iran national Science Foundation (INSF) for the financial support. R. Jafari and S. Tizchang are thankful to the Iran Science Elites Federation for the financial support.
[1] S. L. Glashow, Nucl. Phys. 22, 579 (1961).

[2] S. Weinberg, Phys. Rev. Lett. 19, 1264 (1967).

[3] S. T. Petcov, Sov. J. Nucl. Phys. 25, 340 (1977); ; 25, 1336 (E) (1977)Sov. J. Nucl. Phys.25, 698(E) (1977).

[4] P. Blackstone, M. Fael, and E. Passemar, Eur. Phys. J. C 80, 506 (2020).

[5] J. R. Ellis, J. Hisano, M. Raidal, and Y. Shimizu, Phys. Rev. D 66, 115013 (2002).

[6] P. Paradisi, J. High Energy Phys. 10 (2005) 006.

[7] R. Barbier, C. Berat, M. Besancon, M. Chemtob, A. Deandrea, E. Dudas, P. Fayet, S. Lavignac, G. Moreau, E. Perez et al., Phys. Rep. 420, 1 (2005).

[8] G. Aad et al. (ATLAS Collaboration), Eur. Phys. J. C 76, 232 (2016).

[9] R. Aaij et al. (LHCb Collaboration), J. High Energy Phys. 02 (2015) 121.

[10] G. Aad et al. (ATLAS Collaboration), Eur. Phys. J. C 77, 70 (2017).

[11] R. Aaij et al. (LHCb Collaboration), Phys. Lett. B 754, 167 (2016).

[12] A. M. Sirunyan et al. (CMS Collaboration), J. High Energy Phys. 01 (2021) 163.

[13] G. Aad et al. (ATLAS Collaboration), arXiv:2010.02566.
[14] A. M. Sirunyan et al. (CMS Collaboration), J. High Energy Phys. 03 (2020) 103.

[15] A. M. Baldini et al. (MEG Collaboration), Eur. Phys. J. C 76, 434 (2016).

[16] B. Aubert et al. (BABAR Collaboration), Phys. Rev. Lett. 104, 021802 (2010).

[17] U. Bellgardt et al. (SINDRUM Collaboration), Nucl. Phys. B299, 1 (1988).

[18] J. P. Lees et al. (BABAR Collaboration), Phys. Rev. D 81, 111101 (2010).

[19] K. Hayasaka, K. Inami, Y. Miyazaki, K. Arinstein, V. Aulchenko, T. Aushev, A. M. Bakich, A. Bay, K. Belous, V. Bhardwaj et al., Phys. Lett. B 687, 139 (2010).

[20] E. Kou et al. (Belle-II Collaboration), Prog. Theor. Exp. Phys. 2019, 123C01 (2019); 2020, 029201 (2020).

[21] H. Aihara et al. (ILC Collaboration), arXiv:1901.09829.

[22] H. Baer, T. Barklow, K. Fujii, Y. Gao, A. Hoang, S. Kanemura, J. List, H. E. Logan, A. Nomerotski, M. Perelstein et al., arXiv:1306.6352.

[23] T. Behnke, J. E. Brau, B. Foster, J. Fuster, M. Harrison, J. M. Paterson, M. Peskin, M. Stanitzki, N. Walker, and H. Yamamoto, arXiv:1306.6327. 
[24] P. N. Burrows et al. (CLICdp and CLIC Collaborations), arXiv:1812.06018.

[25] P. Roloff et al. (CLIC and CLICdp Collaborations), arXiv:1812.07986.

[26] M. Aicheler et al. (CLIC Accelerator Collaboration), arXiv:1903.08655.

[27] M. Ahmad, D. Alves, H. An, Q. An, A. Arhrib, N. ArkaniHamed, I. Ahmed, Y. Bai, R. B. Ferroli, Y. Ban et al., IHEPCEPC-DR-2015-01.

[28] IHEP-CEPC-DR-2015-01.

[29] A. Abada et al. (FCC Collaboration), Eur. Phys. J. Special Topics 228, 261 (2019).

[30] J. N. Esteves, J. C. Romao, A. Villanova del Moral, M. Hirsch, J. W. F. Valle, and W. Porod, J. High Energy Phys. 05 (2009) 003.

[31] Y. Cai, T. Han, T. Li, and R. Ruiz, Front. Phys. 6, 40 (2018).

[32] S. Goswami, K. N. Vishnudath, and N. Khan, Phys. Rev. D 99, 075012 (2019).

[33] D. Delepine and F. Vissani, Phys. Lett. B 522, 95 (2001).

[34] J. I. Illana and T. Riemann, Phys. Rev. D 63, 053004 (2001).

[35] A. Flores-Tlalpa, J. M. Hernandez, G. Tavares-Velasco, and J. J. Toscano, Phys. Rev. D 65, 073010 (2002).

[36] M. A. Perez, G. Tavares-Velasco, and J. J. Toscano, Int. J. Mod. Phys. A 19, 159 (2004).

[37] P. S. B. Dev, R. N. Mohapatra, and Y. Zhang, Phys. Rev. Lett. 120, 221804 (2018).

[38] P. S. B. Dev, R. N. Mohapatra, and Y. Zhang, arXiv:1902.04773.

[39] A. Goudelis, O. Lebedev, and J. H. Park, Phys. Lett. B 707, 369 (2012).

[40] A. Vicente, Front. Phys. 7, 174 (2019).

[41] B. Murakami and T. M. P. Tait, Phys. Rev. D 91, 015002 (2015).

[42] G. C. Cho, Y. Fukuda, and T. Kono, Phys. Lett. B 789, 399 (2019).

[43] J. de Favereau, C. Delaere, P. Demin, A. Giammanco, V. Lemaître, A. Mertens, and M. Selvaggi (DELPHES 3 Collaboration), J. High Energy Phys. 02 (2014) 057.

[44] Y. Kuno and Y. Okada, Rev. Mod. Phys. 73, 151 (2001).

[45] M. Tanabashi et al. (Particle Data Group), Phys. Rev. D 98, 030001 (2018).

[46] U. Bellgardt et al. (SINDRUM Collaboration), Nucl. Phys. B299, 1 (1988).

[47] A. M. Baldini et al. (MEG Collaboration), Eur. Phys. J. C 76, 434 (2016).
[48] W. H. Bertl et al. (SINDRUM II Collaboration), Eur. Phys. J. C 47, 337 (2006).

[49] J. Cohen, S. Bar-Shalom, and G. Eilam, Phys. Rev. D 94, 035030 (2016).

[50] H. Khanpour and M. Mohammadi Najafabadi, Phys. Rev. D 95, 055026 (2017).

[51] Y. Afik, S. Bar-Shalom, J. Cohen, and Y. Rozen, Phys. Lett. B 807, 135541 (2020).

[52] Y. Afik, S. Bar-Shalom, A. Soni, and J. Wudka, Phys. Rev. D 103, 075031 (2021).

[53] P. M. Ferreira, R. B. Guedes, and R. Santos, Phys. Rev. D 75, 055015 (2007).

[54] A. Alloul, N. D. Christensen, C. Degrande, C. Duhr, and B. Fuks, Comput. Phys. Commun. 185, 2250 (2014).

[55] C. Degrande, C. Duhr, B. Fuks, D. Grellscheid, O. Mattelaer, and T. Reiter, Comput. Phys. Commun. 183, 1201 (2012).

[56] J. Alwall, M. Herquet, F. Maltoni, O. Mattelaer, and T. Stelzer, J. High Energy Phys. 06 (2011) 128.

[57] J. Alwall, C. Duhr, B. Fuks, O. Mattelaer, D. G. Öztürk, and C. H. Shen, Comput. Phys. Commun. 197, 312 (2015).

[58] J. Alwall, R. Frederix, S. Frixione, V. Hirschi, F. Maltoni, O. Mattelaer, H. S. Shao, T. Stelzer, P. Torrielli, and M. Zaro, J. High Energy Phys. 07 (2014) 079.

[59] C. Chen, Z. Cui, G. Li, Q. Li, M. Ruan, L. Wang, and Q. S. Yan, arXiv:1705.04486.

[60] Q. Li and Q. S. Yan, arXiv:1804.00125.

[61] T. Sjöstrand, S. Ask, J. R. Christiansen, R. Corke, N. Desai, P. Ilten, S. Mrenna, S. Prestel, C. O. Rasmussen, and P.Z. Skands, Comput. Phys. Commun. 191, 159 (2015).

[62] T. Sjostrand, S. Mrenna, and P. Z. Skands, Comput. Phys. Commun. 178, 852 (2008).

[63] H. Abramowicz et al. (ILD Collaboration), arXiv: 1912.04601.

[64] C. T. Potter, arXiv:1602.07748.

[65] A. L. Read, J. Phys. G 28, 2693 (2002).

[66] T. Junk, Nucl. Instrum. Methods Phys. Res., Sect. A 434, 435 (1999).

[67] K. Cranmer et al. (ROOT Collaboration), CERN-OPEN2012-016.

[68] G. Aad et al. (ATLAS and CMS Collaborations), J. High Energy Phys. 08 (2016) 045.

[69] G. Abbiendi et al. (OPAL Collaboration), Phys. Lett. B 519, $23(2001)$ 